\title{
Prevalência de jovens universitários tabagistas e suas inter-relações
}

\section{Prevalence of smoking university students and their interrelationships}

\author{
Victor Barbosa Ribeiro'; Michele Santos de Melo Ireno2; Fábio Luiz Mendonça Martins³; \\ Vanessa Pereira de Lima ${ }^{4}$ \\ ${ }^{1}$ Graduado em Fisioterapia e em Educação Física - UFVJM. Diamantina, MG - Brasil. \\ ${ }^{2}$ Graduada em Fisioterapia - UFVJM. Diamantina, MG - Brasil \\ Professor - UFVJM. Diamantina, MG; Mestre em Fisioterapia - UNITRI. Uberlândia, MG - Brasil. \\ ${ }^{4}$ Professora - UFVJM. Diamantina, MG; Mestre em Ciências da Saúde - UNIFESP, São Paulo, SP - Brasil. \\ Endereço para correspondência \\ Victor Barbosa Ribeiro \\ R. Visconde de Taunay, 748 - apto. 11, Bairro Vila Tibério \\ 14050-500 - Ribeirão Preto - SP [Brasil] \\ victorbarbosa2004@hotmail.com
}

\section{Resumo}

Introdução: $\mathrm{O}$ tabaco é a droga mais consumida no mundo e sua utilização por adolescentes e adultos jovens tem aumentado. Objetivos: Identificar a prevalência do tabagismo em jovens universitários e sua associação com o hábito de consumirem bebidas alcoólicas e de praticarem exercício físico. Método: Foram entrevistados 360 universitários, de ambos os gêneros, idade entre 18 e 24 anos, tendo sido avaliados com relação ao hábito tabágico, nível dependência nicotínica e a outros hábitos associados. Resultados: Encontrou-se uma prevalência de tabagistas de $6,9 \%$, não ocorrendo diferença entre os gêneros. A maioria dos fumantes não realizava exercício físico e apresentava grau baixo e muito baixo de dependência nicotínica. Foi identificada relevante associação entre o hábito de fumar e o consumo de bebidas alcoólicas. Conclusão: É necessário desenvolver programas que incentivem a cessação do hábito tabágico, assim como a divulgação de informações sobre os malefícios do tabaco e bebidas alcoólicas.

Descritores: Adolescente; Estudantes; Tabagismo.

\begin{abstract}
Introduction: Tobacco is the most used drug in the world and its use by teenagers and adults has increased. Objectives: To identify the prevalence of smoking among university students and its association with their habit of consuming alcohol and exercising. Methods: Interviews were conducted with 360 university students of both genders, aged between 18 and 24 years, having been assessed in relation to smoking, nicotine dependence level and other associated habits. Results: The prevalence of smokers was $6.9 \%$, and there was no difference between genders. The majority of smokers did not exercise and had low and very low level of nicotine dependence. It identified significant association between smoking and alcohol consumption. Conclusion: It is necessary to develop programs that encourage tobacco cessation, and the dissemination of information on the dangers of tobacco and alcoholic beverages.
\end{abstract}

Key words: Adolescent; Smoking; Students. 


\section{Introdução}

Segundo o relatório da Organização Mundial da Saúde (OMS) ${ }^{1}$ sobre a epidemia de tabagismo global-2008, o tabaco é a droga mais utilizada no mundo e fator de risco para seis das oito principais causas de morte. Assim, o fumo mata prematuramente de um terço à metade de todas as pessoas que o usam, em média em 15 anos.

Estima-se que cerca de 500 milhões de pessoas, vivas hoje, morrerão como resultado do consumo de tabaco ${ }^{1}$; e, no período de 2002 a 2030, as mortes decorrentes desse uso vão diminuir em 9\% nos países desenvolvidos, mas aumentar em 100\% (para 6,8 milhões), nos países em desenvolvimento ${ }^{2}$.

Os prejuízos causados à saúde pelo hábito de fumar são discutidos e seu controle é considerado pela OMS como um dos maiores desafios da saúde pública atualmente ${ }^{3}$. O tabagismo, em qualquer uma de suas formas, está relacionado com $90 \%$ de todos os cânceres de pulmão e é um fator de risco para acidentes cerebrovasculares e ataques cardíacos fatais ${ }^{1}$.

Aproximadamente $80 \%$ dos fumantes mostram interesse em parar de fumar; entretanto, apenas $3 \%$, a cada ano, conseguem deixar o fumo, e desses indivíduos, somente $2,7 \%$ param sem um tratamento formal ${ }^{4}$.

Sabe-se que a dependência nicotínica pode ser a chave da persistência do tabagismo e da dificuldade de sua suspensão e que, possivelmente, todos os fumantes regulares são dependentes dessa substância, ainda que em graus variáveis ${ }^{5}$.

A cada dia, cerca de 100 mil jovens começam a fumar, e $80 \%$ se encontram em países em desenvolvimento ${ }^{1}$. A idade média da iniciação ao fumo do tabaco é de 15 anos, o que levou a OMS a considerar o tabagismo com uma doença pediátrica. Também se evidencia aumento da prevalência do uso de cigarros entre a população de adolescentes e adultos jovens ${ }^{6}$, principalmente entre os estudantes universitários jovens ${ }^{7}$, público esse com grande susceptibilidade de envolvimento com o tabaco ${ }^{6}$.
Alguns autores relataram associação entre sedentarismo e tabagismo ${ }^{8}$, outros que ocorre uma redução da morbimortalidade quando fumantes passam a ser ativos fisicamente ${ }^{9},{ }^{10}$. Já outros pesquisadores observaram que adolescentes tabagistas apresentaram menores níveis de atividade física, o que, no período da adolescência, leva ao início do hábito tabágico e em sua manutenção na idade adulta ${ }^{11}$.

Em outro estudo ${ }^{12}$, afirma-se que tabagistas também apresentaram maior proporção e intensidade de consumo de bebida alcoólica, sendo significante a diferença, quando comparados aos ex-tabagistas.

Como os malefícios causados pelo tabaco são muito conhecidos, esperava-se que os estudantes de nível superior (universitários) tivessem melhores informações a esse respeito e assim evitassem adquirir esse hábito nocivo. Entretanto, estudos apontam uma alta prevalência do uso de cigarros na população de adolescentes e adultos jovens, principalmente entre os estudantes universitários ${ }^{6,13}$.

Dessa forma, o objetivo neste estudo é identificar a prevalência de tabagismo em jovens universitários, a diferença de uso entre gêneros, bem como o início do hábito de fumar, sua relação com o nível de dependência nicotínica, sua associação com a prática de atividade física e o consumo de bebidas alcoólicas.

\section{Material e métodos}

\section{Local e tipo de estudo}

Este estudo foi do tipo transversal. Toda a anamnese e questionários foram aplicados nas dependências da Universidade Federal dos Vales do Jequitinhonha e Mucuri (UFVJM), campus Diamantina.

\section{Aspectos éticos}

A pesquisa foi conduzida de acordo com a Resolução 196 de 1996 do Conselho Nacional de Saúde e foi executada após a aprovação pelo 
Comitê de Ética em Pesquisa da UFVJM. Ao apresentarem-se como voluntários, os indivíduos foram informados pelos pesquisadores quanto aos objetivos e procedimentos metodológicos do estudo. O consentimento para participação no trabalho foi obtido por escrito de cada voluntário, após os esclarecimentos necessários, estando todos cientes de que a qualquer momento poderiam, sem constrangimento, deixar de participar do estudo. Foram tomadas todas as precauções no intuito de preservar a privacidade dos voluntários.

\section{Amostra}

Para o cálculo do tamanho da amostra, foi admitido como estimativa de proporção " $p$ " de tabagistas entre 18 e 24 anos. Utilizou-se a porcentagem de $29,4 \%$ de tabagistas como base de comparação para este estudo, sendo esse dado obtido em estudo prévio realizado, em 2001, pelo Centro Brasileiro de Informações sobre Drogas Psicotrópicas ${ }^{14}$. Já o erro alfa considerado foi $3 \%$, sendo a precisão desejada em torno da prevalência, para possibilitar intervalo de confiança de $95 \%{ }^{15}$. Foram acrescidos 16 estudantes no cálculo encontrado de 344 universitários da UFVJM, no campus de Diamantina, para facilitar o sorteio e a divisão dos entrevistados. Desta maneira, obteve-se uma amostra total de 360 voluntários selecionados. A Universidade, no momento da seleção dos voluntários, oferecia 13 cursos com pelo menos uma turma graduada, que serviu como critério de inclusão dos cursos no sorteio, sendo seis sorteados aleatoriamente para compor a amostra ${ }^{15}$ (Agronomia, Zootecnia, Sistemas de Informação, Química, Educação Física, Farmácia). Foram sorteados 60 voluntários de cada um dos seis cursos, totalizando 360 voluntários. Dos 60 voluntários de cada curso, como critério de comparação, 30 necessariamente deveriam estar matriculados no início do curso (nos três primeiros períodos); e 30, no final (nos três últimos períodos de cada curso que estivessem presentes na cidade).
Os critérios de inclusão para o estudo foram: estar matriculado na Universidade Federal dos Vales do Jequitinhonha e Mucuri em um dos cursos acima citados; estar na sala de aula no momento da visita; ter entre 18 e 24 anos e a aceitar os termos de consentimento livre e esclarecido.

\section{Procedimentos}

\section{Relação de entrevistados e aplicação dos questionórios}

Após o sorteio dos cursos, foram programadas as datas e os horários de visitas a cada turma, que ocorreram uma vez durante o momento da aula. Na data programada, foram realizados esclarecimentos e após aceitar as condições do estudo o voluntário assinava o termo de compromisso livre e esclarecido. Posteriormente, o participante recebia a folha de anamnese em que constavam os campos de identificação (nome, endereço e telefone), de preenchimento opcional, e outros campos de informações (idade, sexo, período e curso) os quais era necessário preencher, além de perguntas básicas sobre hábitos de vida, tais como consumo de bebidas alcoólicas, realização de exercício físico, período em que começaram a fumar (antes ou após o ingresso na universidade) e hábito tabágico. Caso fossem tabagistas, os voluntários eram questionados quanto ao interesse em participar de um programa de cessação tabágica.

Para ser classificado como consumidor de bebidas alcoólicas, o voluntário deveria ingerilas pelo menos uma vez por semana. Já com relação à prática de exercício físico, o American College of Sports Medicine e o American Heart Association preconiza que para manter a saúde, todos os jovens e adultos entre 18 e 65 anos, precisam realizar atividade física de intensidade aeróbia por um período mínimo de 30 minutos durante cinco dias por semana, ou de intensidade vigorosa, por no mínimo 20 minutos, por três dias na semana ${ }^{16}$. Neste estudo, considerou-se praticante de um programa de exercício físico, o indivíduo que executasse de forma programa- 
da pelo menos 30 minutos de atividade física, por três dias na semana. Os estudantes tabagistas foram avaliados quanto ao grau de dependência nicotínica por meio do questionário de tolerância de Fargeström $(\mathrm{QTF})^{5}$. Classificou-se tabagista, o sujeito que fizesse uso do tabaco diariamente e que possuísse pelo menos o grau muito baixo de dependência nicotínica, como definido por esse questionário. Os voluntários tabagistas receberam no final do estudo, orientações por meio de cartilhas sobre o tabagismo. Os universitários que manifestaram interesse em participar de outros programas de educação em saúde que visam à cessação tabágica foram cadastrados em um banco de dados digital de fins acadêmicos para que possam ser chamados para futuros programas.

\section{Anólise estatística}

Foram realizadas análises descritivas (frequência) e as associações entre as variáveis dependentes (fumante e dependência nicotínica). Também foram testadas as variáveis independentes por meio de análise univariada (teste do qui-quadrado), sendo considerado o valor de $p<0,05$ para significância estatística bem como o teste exato de Fisher.

\section{Resultados}

Dos 360 entrevistados, 50,6\% eram acadêmicos do gênero masculino; e 49,4\%, do feminino, com média de idade de 21,2 anos (Tabela 1).

Verificou-se prevalência de $6,9 \%$ de tabagistas na população geral estudada, sendo $4,7 \%$ homens; e 2,2\%, mulheres. Considerando-se apenas o gênero masculino, 9,3\%, eram fumantes; e levando-se em conta somente o feminino, $4,5 \%$ eram tabagistas. Não foi encontrada diferença estatística no consumo do tabaco entre os gêneros ( $p=0,071)$, nem quando se relacionou o gênero do voluntário tabagista com os diferentes períodos $(\mathrm{p}=0,186)$ (Tabela 1$)$.
Tabela l: Prevalência do tabagismo entre os gêneros nos períodos iniciais e finais do curso e prevalência do tabagismo entre os gêneros

\begin{tabular}{cccc}
\hline \multirow{2}{*}{ Amostra } & \multicolumn{2}{c}{ Gênero } & \multirow{2}{*}{ Total } \\
& Masculino & Feminino & \\
\hline $\begin{array}{c}\text { Tabagistas - } \\
\text { períodos iniciais }\end{array}$ & 4 & 4 & 8 \\
$\begin{array}{c}\text { Tabagistas - } \\
\text { períodos finais }\end{array}$ & 13 & 4 & 17 \\
Tabagistas & 17 & 8 & 25 \\
Não tabagistas & 165 & 170 & 335 \\
Total & 182 & 178 & 360 \\
\hline
\end{tabular}

*Todos os valores não foram estatisticamente significativos.

Quanto ao nível de dependência nicotínica, $72 \%$ dos universitários tabagistas possuíam muito baixa ou baixa dependência nicotínica, enquanto apenas $28 \%$ estavam com média ou alta dependência nicotínica. Dos 25 universitários tabagistas, apenas $28 \%$ (sete tabagistas) relataram ter começado a fumar após a entrada na Universidade, e destes, cinco estavam matriculados nos períodos finais.

Quando questionados sobre o interesse em participar de um programa de cessação tabágica, $52 \%$ dos entrevistados demonstraram interesse, enquanto $48 \%$ não apresentaram.

Tratando-se da relação tabagismo x prática de exercício físico programado (Tabela 2), não foi encontrada diferença estatisticamente significativa ( $p=0,088)$, não havendo diferença quanto à prática de exercício físico entre a população tabagista e não tabagista, assim como, quando se comparou fumantes nos períodos iniciais dos cursos, com os dos finais, quanto à prática de exercício físico $(\mathrm{p}=0,127)$ (Tabela 2).

Com relação ao consumo de bebidas alcoólicas, quando comparados indivíduos tabagistas e não tabagistas, foi encontrado que 100,0\% da população consomem bebidas alcoólicas. Quando comparado o consumo de bebidas alcoólicas entre os períodos iniciais e finais dos cursos, nota-se um consumo maior de bebidas alcoólicas nos períodos finais dos cursos de graduação $(p=0,003)$ (Tabela 3). 
Tabela 2: Relação da prótica de exercício físico dos tabagistas dos períodos iniciais e finais e Relação da prótica de exercício pelos tabagistas e não tabagistas

\begin{tabular}{cccc} 
Amostra & \multicolumn{2}{c}{$\begin{array}{c}\text { Exercício físico } \\
\text { regular }\end{array}$} & Total \\
Tabagista - & Sim & Não & \\
períodos iniciais & 2 & 6 & 8 \\
Tabagistas - & & & \\
períodos finais & 8 & 9 & 17 \\
Tabagistas & 10 & 15 & 25 \\
Não tabagistas & 87 & 248 & 335 \\
$\quad$ Total & 97 & 263 & 360
\end{tabular}

*Todos os valores não foram estatisticamente significativos.

Tabela 3: Relação do consumo de bebidas alcoólicas pelos tabagistas e relação do consumo de bebidas alcoólicas entre os estudantes dos períodos iniciais e finais dos cursos

\begin{tabular}{|c|c|c|c|}
\hline \multirow[t]{2}{*}{ Amostra } & \multicolumn{2}{|c|}{$\begin{array}{c}\text { Consumo de bebidas } \\
\text { alcoólicas }\end{array}$} & \multirow[t]{2}{*}{ Total } \\
\hline & Sim & Não & \\
\hline Tabagistas* & 25 & 0 & 25 \\
\hline Não tabagistas & 202 & 133 & 335 \\
\hline Total & 227 & 133 & 360 \\
\hline $\begin{array}{l}\text { Estudantes - } \\
\text { períodos iniciais }\end{array}$ & 100 & 80 & 180 \\
\hline $\begin{array}{l}\text { Estudantes - } \\
\text { períodos finais }\end{array}$ & $127^{*}$ & 53 & 180 \\
\hline
\end{tabular}

\section{Discussão}

Vários estudos vêm sendo realizados com a intenção de avaliar a prevalência de tabagismo e maus hábitos de vida em populações mais jovens $^{3,6,7,8,11}$. No estudo aqui apresentado, objetivou-se avaliar a relação entre o hábito de fumar, o consumo de bebidas alcoólicas e a inatividade física em jovens universitários, bem como identificar diferentes prevalências do tabagismo entre gêneros, nível de dependência nicotínica dos tabagistas e o interesse em participar de programas de cessação tabágica.

Analisando a prevalência de tabagismo em jovens da Universidade Rei Saud, na Arábia Saudita, com idade entre 17 e 25 anos $^{17}$, foi encontrada a prevalência de $14,5 \%$ de fumantes. Segundo o I levantamento nacional sobre o uso de álcool, tabaco e outras drogas entre universitários das 27 capitais brasileiras ${ }^{18}$, realizado no ano de 2010 pela secretária nacional de políticas sobre drogas, $19,1 \%$ dos universitários na faixa etária entre 18 e 24 anos, haviam feito uso de algum produto do tabaco nos últimos 30 dias à entrevista. Possivelmente, a diferença dos percentuais entre os estudos, deve-se ao fato de que neste estudo avaliaram-se apenas os indivíduos consumidores diários do tabaco, não levando em consideração os eventuais ou que já consumiram em algum momento da sua vida. Nos dados deste estudo, apenas 6,9\% dos universitários entrevistados afirmaram fazer uso contínuo de substâncias ligadas ao tabaco. Em estudo realizado em Brasília, encontrou-se uma prevalência de tabagismo regular de $9 \%$ entre a faixa etária de 18 a 24 anos em jovens universitários, corroborando os dados encontrados neste trabalho ${ }^{15}$.

Com relação ao consumo por gênero, foi encontrada, por outros autores ${ }^{8}$, uma maior prevalência de tabagistas do sexo feminino em relação ao masculino. No entanto, vários trabalhos têm identificado uma maior prevalência de consumo por indivíduos do sexo masculino ${ }^{19-22}$. Em estudantes do ensino médio foi obtida uma prevalência numericamente maior de uso do tabaco por indivíduos do sexo masculino, compondo $63,6 \%$ dos indivíduos fumantes, enquanto a amostra do sexo feminino era composta apenas por $36,4^{19}$. Esses resultados são semelhantes aos encontrados neste estudo, no qual $68 \%$ da amostra entrevistada que fazia consumo de tabaco foi formada por homens jovens, enquanto os outros 32\% eram mulheres jovens. Entretanto, assim como o encontrado por outros estudos $^{21,22}$ não houve diferença estatisticamente significativa entre o consumo de tabaco por jovens de ambos os gêneros. 
A investigação entre inatividade física e o hábito de fumar vêm sendo elucidada; porém, ainda não há dados que relacionem a inatividade física e o tabagismo ${ }^{23}$, resultado semelhante ao encontrado na atual investigação.

Outro ponto que merece destaque é que a maior parte dos jovens tabagistas deste estudo $(76 \%)$ já consumia tabaco antes do acesso à universidade, e haviam iniciado esse hábito com idade inferior a 19 anos, semelhante ao constatado em outro estudo ${ }^{24}$ em que se identificou alta prevalência do hábito tabágico em jovens com idade inferior a 20 anos. Dessa forma, sugerese que há necessidade de cuidados preventivos contra o fumo em idades mais precoces.

No atual estudo, não foi verificado o consumo de tabaco esporadicamente, o que poderia elevar consideravelmente o número de consumidores de fumo como encontrado em outra investigação ${ }^{25}$. No trabalho aqui apresentado, também se identificou uma relação estatisticamente significativa entre tabagismo e abuso no consumo de álcool.

Em uma pesquisa envolvendo as características do consumo de bebidas alcoólicas e tabagismo em estudantes da Faculdade de Medicina da Universidade Federal de Uberlândia, levantou-se a hipótese de uma possível associação entre o hábito de fumar e o consumo de bebida alcoólica, pois os jovens apresentaram idade de início do hábito de fumar semelhante a que iniciaram o consumo de bebidas alcoólicas ${ }^{26}$. Outros autores ${ }^{20,21}$ também encontraram o predomínio de jovens que faziam uso do cigarro associado à bebida alcoólica, estando de acordo com este estudo em que se verificou que todos jovens tabagistas faziam o uso de bebida alcoólica.

Quanto ao grau de dependência nicotínica, no atual estudo, $72 \%$ dos fumantes apresentaram baixo grau de dependência nicotínica, o que difere dos dados encontrados em uma pesquisa feita com tabagistas regulares, adultos (maiores de 15 anos, com média de idade de 48,6 anos) que apresentou $54,9 \%$ dos fumantes pertencentes ao grupo de elevada dependência nicotínica no questionário de Fagerström ( $\geq 6$ pontos $)^{5}$.
Apesar de todos os danos causados pelo hábito de fumar, apenas $52 \%$ dos indivíduos encontrados como tabagistas no atual estudo demonstraram interesse em participar de um programa de cessação tabágica. Resultado semelhante foi obtido em um estudo transversal de prevalência e características do tabagismo em alunos da Universidade de Brasília (UnB), no qual $68 \%$ dos entrevistados relataram a intenção de deixar de fumar ${ }^{15}$.

Ao avaliar as diferenças entre gênero, quantidade de tabagistas, fumantes que consumiam bebidas alcoólicas e os que realizavam atividade física, não foram encontradas diferenças estatisticamente significativas com relação ao fato de os universitários estarem nos períodos iniciais ou finais do seu curso. Também vale a pena ressaltar que neste estudo não foi avaliada a quantidade de estudantes que fazem uso do tabaco esporadicamente, o que poderia aumentar o número da população tabagista e talvez gerar uma diferença entre os períodos iniciais e finais, já que a maior parte da população que consome bebidas alcoólicas encontra-se nos períodos finais do seu curso, sugerindo que há uma possível relação entre tabagistas esporádicos e consumo de bebidas alcoólicas como encontrado por outros pesquisadores ${ }^{25}$. Outro ponto que não foi avaliado foi o tipo do exercício realizado (aeróbio e anaeróbio) e sua intensidade, o que poderia gerar outros resultados.

Por fim, é importante que seja levantada a hipótese de que resultados diferenciados poderiam ser encontrados em outras instituições. Assim, os dados aqui obtidos, talvez não tenham como serem comparados aos de outros locais, como em instituições privadas, que, muitas vezes, têm outro perfil social. Logo, essa diferença também poderia, consequentemente, gerar hábitos e resultados diferentes.

\section{Conclusão}

Conclui-se que, na população estudada, a prevalência de tabagismo foi baixa, quando 
comparada a outros estudos, principalmente por não se ter avaliados os tabagistas esporádicos. Também foi verificado um baixo nível de dependência nicotínica na maior parte dos tabagistas, além disso, constatou-se que quase metade deles não tem interesse em participar de um programa de cessação tabágica. Esse resultado não era esperado, levando em consideração o nível de instrução da população estudada. Com relação à prática de exercício físico, não foi encontrado número de praticantes expressivo em nenhum dos grupos estudados, dado esse preocupante, uma vez que essa atividade é considerada como uma incentivadora para mudança de maus hábitos de vida. Quanto ao consumo de bebidas alcoólicas, os tabagistas mostraram um número superior de consumo dessas bebidas, quando comparado ao dos indivíduos não tabagistas. Diante do exposto, salienta-se a necessidade da criação de programas preventivos de educação em saúde que visem o incentivo à cessação tabágica e a divulgação de informações sobre os malefícios causados tanto pelo tabagismo quanto pelo consumo de bebidas alcoólicas, além de acompanhar o comportamento dos universitários após o término da graduação com relação ao hábito tabágico. Dessa forma, é importante que novos estudos sejam realizados para reforçar os achados deste estudo.

\section{Referências}

1. Organização Mundial de Saúde. Relatório da OMS sobre a epidemia global de tabagismo, 2008: Pacote MPOWE. [acesso em 2010 fev 28]. Disponível em: $<$ www.who.int/tabaco/mpower $>$.

2. Oliveira AF, Valente JG, Leite IC. Aspectos da mortalidade atribuível ao tabaco: revisão sistemática. Rev Saúde Pública. 2008;42(2);335-45.

3. Horta BL, Calheiros P, Pinheiro RT, Tomasi E, Amaral KC. Tabagismo em adolescentes de área urbana na região sul do Brasil. Rev Saúde Pública. 2001;35(2);159-64.
4. Silva AO, Sousa CMM, Gaspar MFM, Paredes MAS, Tura LFR, Jesuíno JC. Tabaco e saúde no olhar de estudantes universitários. Rev Bras Enferm. 2008;61(4);423-7.

5. Halty LH, Hutiner MD, Netto ICO, Santos VA, Martins G. Análise da utilização do Questionário de Tolerância de Fagerström (QTF) como instrumento de medida da dependência nicotínica. J Pneumol. 2002;28(4);180-6.

6. Rigotti NA, Lee JE, Wechsler H. US college students' use of tobacco products: results of a national survey. JAMA. 2000;284(6);699-705.

7. Willcox ML. Tobacco control programs for universities: a feasibility study. J Public Health Med. 1997;19(1);37-44.

8. Holmen TL, Connor EB, Clausen J, Holmen J, Bjermer L. Physical exercise, sports, and lung function in smoking versus nonsmoking adolescents. Eur Respir J. 2002;19;8-15.

9. Colbert LH, Hartman TJ, Malila N, Limburg PJ, Pietinen P, Virtamo J. Physical activity in relation to cancer of the colon and rectum in a cohort of male smokers. Cancer Epidemiol Biomarkers Prev. 2011;10(3);265-8.

10. Colbert LH, Hartman TJ, Tangrea JA, Pietinen P, Virtamo, J, Taylor R. Physical activity and lung cancer risk in male smokers. Int J Cancer. 2002;98(5);770-3.

11. Higgins JW, Gaul C, Gibbons S, Gyn GV. Factors influencing physical activity levels among Canadian youth. Can J Public Health. 2003;94(1);45-51.

12. Lemos-Santos MGF, Valente JG, Gonçalves-Silva RMV, Botelho C. Relação entre tabagismo e outras características do estilo de vida de uma população adulta, Cuiabá-MT. J Bras Pneumol. 2001;23(sp. Issue), tl 1.

13. Hallal ALC, Gotlieb SLD, Almeida LM, Casado L. Prevalência e fatores associados ao tabagismo em escolares da região Sul do Brasil. Rev Saúde Pública. 2009;43(5);779-88.

14. Carlini EA, Galduróz JCF, Noto AR, Nappo SA. I Levantamento domiciliar sobre o uso de drogas psicotrópicas no Brasil: estudo envolvendo as 107 maiores cidades do país - 2001. São Paulo: Centro Brasileiro de Informações sobre Drogas Psicotrópicas (CEBRID); 2002. 
15. Andrade APA, Bernardo ACC, Viegas CAA, Ferreira DBL, Gomes TC, Sales MR. Prevalência e características do tabagismo em jovens da Universidade de Brasília. J Bras Pneumol. 2006;32(1);23-8.

16. Haskell WL, Lee IM, Pate RR, Powell KE, Blair SN, Franklin BA, et al. Physical activity and public health. Updated recommendation for adults from the American College of Sports Medicine and the American Heart Association. Circulation. 2007 [Epub doi: 10.1161/circulationaha.107.185649].

17. Mandil A, Binsaeed A, Ahmad S, Al-dabbagh R, Alsaadi M, Khan M. Smoking among university students: a gender analysis. J Infect Public Health. 2010;3;179-87.

18. Andrade AG, Duarte PCAV, Oliveira LG. I Levantamento nacional sobre o uso de álcool, tabaco e outras drogas entre universitários das 27 capitais brasileiras. Secretaria nacional de políticas sobre drogas, 285 p.; 2010.

19. Tamaki T, Kaneita Y, Ohida T, Yokoyama E, Osaki Y, Kanda H. et al. Prevalence of and factors associated with smoking among Japanese medical students. J Epidemiol. 2010;20(4):339-45.

20. Almeida AF, Mussi FC. Tabagismo: conhecimentos, atitudes, hábitos e grau de dependência de jovens fumantes em Salvador*. Rev Esc Enferm USP. 2006;40(4):456-63.
21. Ferreira MMSRS, Torgal MC LFPR. Tobacco and alcohol consumption among adolescents. Rev Latino-Am Enferm. 2010;18(2):255-61.

22. Moreno RS, Ventura RN, Brêtas JRS. O uso de álcool e tabaco por adolescentes do município de Embu, São Paulo, Brasil. Rev Esc Enferm USP. 2010;44(4):969-77.

23. Rodrigues ESR, Cheik NC, Mayer AF. Nível de atividade física e tabagismo em universitários. Rev Saúde Pública. 2008;42(4):672-8.

24. Malcon MC, Menezes AMB, Chatkin M. Prevalência e fatores de risco para tabagismo em adolescentes. Rev Saúde Pública. 2003;37(1):1-7.

25. Rocha MIUM, Barrio-lera JP, Jardim GBH, Mucellini $\mathrm{AB}$, Cirolini L, Jung IEC, et al. Estilo de vida, características de saúde e consumo excessivo de álcool em adultos jovens que são fumantes esporádicos. São Paulo Med J. 2010;128(6);354-9.

26. Paduani GF, Barbosa GA, Morais JCR, Pereira JCP, Almeida MF, Prado MM, et al. Consumo de álcool e fumo entre os estudantes da Faculdade de Medicina da Universidade Federal de Uberlândia. Rev Bras Educ Méd. 2008;32(1);66-75. 